Canad. Math. Bull. Vol. 22 (4), 1979

\title{
ON QUOTIENT LOOPS OF NORMAL SUBLOOPS
}

\author{
BY \\ C. SANTHAKUMARI
}

1. Introduction. The following result is due to Wielandt [1, Lemma 2.9]: Let $A, B, K$ be $N$-submodules of some $N$-module, where $N$ is a zero symmetric near-ring. Then the $N$-module, $\Gamma:=(A+K) \cap(B+K) \mid(A \cap B)+K$ is commutative. Using this result Wielandt obtained density theorem for 2-primitive near-rings with identity. Betsch [1] used Wielandt's result to obtain the density theorem for 0 -primitive near-rings. The purpose of this paper is to extend this result for loops.

2. Result. We prove the result for additive loops. For the definitions of loops, normal subloops see [2]. If $G$ is any additive loop and $H$ is a normal subloop of $G$, the quotient loop $G$ modulo $H$ is denoted by $G / H$ in which addition is defined as $(x+H)+(y+H)=(x+y)+H$ for all $x+H, y+H$ in $G / H$ [2, p. 61]; further, $x \in H$ iff $x+H=H$. Let $G$ be any additive loop. For any $a \in G$, we shall denote the unique left and right additive inverses of $a$ by $a_{1}$ and $a_{r}$ respectively.

Proposition 2.1. Let $G$ be an additive loop and $A, B, K$ be normal subloops of $G$, then the additive loop $\bar{G}=(A+K) \cap(B+K) \mid(A \cap B)+K$ is an additive abelian group.

Proof. Let $E=(A \cap B)+K$ and $H=(A+K) \cap(B+K)$ and let 0 be the identity of the loop $G$. It is enough to show that for all $x, y, z \in H$; $(x+(y+z))+E=((x+y)+z)+E$ and $(x+y)+E=(y+x)+E$. Let $x, y, z \in H$. Then, $x \in A+K$; hence $x=a+p$, for some $a \in A$ and $p \in K$. Since, $y, z \in H$; $y, z \in B+K$, hence, $y=b+q$ and $z=c+r$, where $b, c \in B$ and $q, r \in K$. We wish to show that $x+E=a+E, y+E=b+E$ and $z+E=c+E$. Since $E$ is a normal subloop of $G$ [2, iv, Theorem 1.2 and Theorem 1.4], $(x+E)=(a+p)+E=$ $a+(p+E)$. Since $p \in K, p \in E$ and hence $p+E=E$. Therefore, $x+E=a+E$. By a similar argument we get, $y+E=b+E$ and $z+E=c+E$. Since $A$ is a normal subloop of $G$ and since $a \in A, A+((a+b)+c)=(A+(a+b))+c=$ $((A+a)+b)+c=(A+b)+c=A+(b+c)$ and $A+(a+(b+c))=(A+a)+$ $(b+c)=A+(b+c)$. Therefore, $A+((a+b)+c)=A+(a+(b+c))$. Since, $(a+b)+c \in A+((a+b)+c)$, we have $(a+b)+c \in A+(a+(b+c))$; since $A$ is a normal subloop of $G$, we have,

$$
\begin{aligned}
((a+b)+c)+(a+(b+c))_{r} \in\{A+ & (a+(b+c))\}+(a+(b+c))_{r} \\
= & A+\left\{(a+(b+c))+(a+(b+c))_{r}\right\}=A+0=A .
\end{aligned}
$$

Received by the editors March 2, 1978 and, in revised form, October 2, 1978. 
By a similar argument, we get, $B+((a+b)+c)=B+(a+(b+c))$ and consequently $((a+b)+c)+(a+(b+c))_{r} \in B$. Therefore, $\quad((a+b)+c)+(a+$ $(b+c))_{r} \in A \cap B \subseteq E$. Hence,

$$
\left\{((a+b)+c)+(a+(b+c))_{r}\right\}+E=E=0+E=\left\{(a+(b+c))+(a+(b+c))_{r}\right\}+E .
$$

Since $G / E$ is a loop and since cancellation laws hold in a loop, we get, $(a+(b+c))+E=((a+b)+c)+E$. Therefore,

$$
(x+(y+z))+E=(a+(b+c))+E=((a+b)+c)+E=((x+y)+z)+E .
$$

Hence, the loop $\bar{G}$ is associative and consequently $\bar{G}$ is a group. Now we show that $\bar{G}$ is abelian. Since $A$ is a normal subloop of $G$ and $a \in A$ we have, $A+(a+b)=(A+a)+b=A+b$ and $A+(b+a)=(b+a)+A=b+(a+A)=$ $b+A=A+b$. Therefore, $A+(a+b)=A+(b+a)$ and hence, $(a+b)+$ $(b+a)_{r} \in A$. By a similar argument we get, $(a+b)+(b+a)_{r} \in B$. Therefore $(a+b)+(b+a)_{r} \in A \cap B \subseteq E$. Hence, $\quad(a+b)+E=(b+a)+E$. Therefore, $(x+y)+E=(a+b)+E=(b+a)+E=(y+x)+E$. Hence, $\bar{G}$ is abelian. Hence the result.

Corollary 2.2. Let $G$ be an additive loop and let no nonzero epimorphic image of any normal subloop of $G$ be an abelian group. Then the lattice of normal subloops of $G$ is distributive.

Proof. Let $A, B, K$ be normal subloops of $G$ and let $H=(A+K) \cap(B+K)$, $E=(A \cap B)+K$ and $\bar{G}=H / E$. Now, $H$ is a normal subloop of $G[2$, iv, Theorems $1.2 ; 1.4], \bar{G}$ is an epimorphic image of $H$; but $\bar{G}$ is an abelian group (prop. 2.1), hence we must have $H=E$, that is, $(A+K) \cap(B+K)=$ $(A \cap B)+K$. Therefore, the lattice of normal subloops of $G$ is distributive.

ACKnowledgement. I wish to thank the referee and Professor D. Ramakotaiah for their valuable suggestions. I would also like to thank the Council of Scientific and Industrial Research, New Delhi, India, for the financial assistance.

\title{
REFERENCES
}

1. G. Betsch, Primitive near-rings, Math. z, 130, 351-461 (1973).

2. R. Hubert Bruck, A survey of binary systems, Springer-Verlag, New York, Inc, 1966.

\author{
DepartMent of Mathematics \\ NAGARJUNA UNIVERSITY \\ NAGARJUNANAGAR, 522510 \\ ANDHRA PRADESH, INDIA.
}

